\title{
Mechanical behaviour and rupture of normal and pathological human ascending aortic wall
}

\author{
C. M. García-Herrera • J. M. Atienza • F. J. Rojo • \\ E. Claes · G. V. Guinea $\cdot$ D. J. Celentano · \\ C. García-Montero · R. L. Burgos
}

\begin{abstract}
The mechanical properties of aortic wall, both healthy and pathological, are needed in order to develop and improve diagnostic and interventional criteria, and for the development of mechanical models to assess arterial integrity. This study focuses on the mechanical behaviour and rupture conditions of the human ascending aorta and its relationship with age and pathologies. Fresh ascending aortic specimens harvested from 23 healthy donors, 12 patients with bicuspid aortic valve (BAV) and 14 with aneurysm were tensile-tested in vitro under physiological conditions. Tensile strength, stretch at failure and elbow stress were measured. The obtained results showed that age causes a major reduction in the mechanical parameters of healthy ascending aortic tissue, and that no significant differences are found between the mechanical strength of aneurysmal or BAV aortic specimens and the corresponding age-matched control group. The physiological level of
\end{abstract}

the stress in the circumferential direction was also computed to assess the physiological operation range of healthy and diseased ascending aortas. The mean physiological wall stress acting on pathologic aortas was found to be far from rupture, with factors of safety (defined as the ratio of tensile strength to the mean wall stress) larger than six. In contrast, the physiological operation of pathologic vessels lays in the stiff part of the response curve, losing part of its function of damping the pressure waves from the heart.

Keywords Mechanical properties - Tensile strength . Aneurysm - Bicuspid aortic valve

\section{Introduction}

The aorta is the paradigm of major arteries, which not only serve as conduits for the bloodstreams but they also play a key role in modulating pressure and flow in the entire circulatory system by means of their mechanical response. Major vascular diseases alter and degrade the structure and composition of the aortic wall, and have an effect on its mechanical behaviour. Consequently, a better understanding of the mechanical performance of the vessel wall and its connection with pathologies should be warranted in order to prevent circulatory accidents, as well as for the development of effective and reliable treatments and surgical procedures $[2,20]$.

Aneurysms are placed among the severe, potentially life-threatening abnormalities of the aorta. They are caused by a pathological expansion of the aortic diameter, which may lead to dissection and rupture. Interventional criteria balance the risks associated with surgical repair with the risk of complications due to the development of the disease. The risk of growth and rupture is commonly related 
to aortic diameter, with this being the most used criterion for intervention [1,7]. Although there is considerable evidence that the risk of rupture, dissection or death is dramatically increased in thoracic aortic aneurysms with diameters in excess of 60 or $70 \mathrm{~mm}$ (for ascending or descending aorta, respectively) [3,5], the fraction of aneurysms that rupture before reaching that size is not negligible $[4,14,24]$. Rupture of aneurysms occurs when the mechanical stresses acting on that zone of the vessel exceed the strength of the wall tissue. It seems that a particular patient-specific criterion is therefore necessary. However, all the size-based criteria, either considering absolute [1] or relative aortic diameters [6], do not take into account explicitly the actual mechanical behaviour of the arterial wall. Enhanced and more reliable criteria accounting for the particular characteristics of every patient, and more closely related to the mechanical performance and strength of the arterial wall, are still needed.

Customarily associated to aortic disease and aneurysm development, bicuspid aortic valve (BAV) is an anomaly probably due to the presence of a genetic defect that, in addition to valve leaflets, affects the medial layer of aorta [8]. The high risk of dilatation and aortic dissection-up to nine times higher-favours that many asymptomatic patients are operated on prophylactically for aortic replacement [1]. Nevertheless, recent studies have questioned such a practice on the basis that surgical guidelines are not based on objective evidence [12].

The accumulated experience with aneurysmal and BAV patients - two prevalent aortic pathologies - demonstrates the relevance of obtaining experimental information on the mechanical behaviour and strength of the pathological aorta, in order to develop accurate and dependable diagnostic and interventional criteria.

Nevertheless, data on the mechanical strength of human ascending aorta are still limited and somehow controversial. It is generally agreed that healthy tissue is stronger than aneurysmal in both longitudinal and circumferential directions. Control and aneurysmal ascending aorta in both such orientations have been compared, with findings supporting this contention [23]. Pathologic tissue was $30 \%$ weaker and appreciably stiffer than control. However, other authors presented the unexpected finding of aneurysmal aorta as being as equally resistant as normal aortic tissue, and pointed out the importance of considering agematched subject groups before drawing conclusions [16]. In particular, these authors tested aneurysm tissue from four regions and reported an increased stiffness and reduction in extensibility compared to control tissue and anisotropy of both aneurysm and control tissue. The influence of age on the rupture of pathological ascending aorta and aneurysmal strengths in between the other two studies have been analysed and reported [19]. The fact that most of the few available studies on the tensile properties of the ascending aortic wall $[19,23]$ have been performed at a non-physiological temperature $\left(\sim 20^{\circ} \mathrm{C}\right)$, and the variable nature of most control vessels that were taken from autopsies, might be at the root of these differences. It was demonstrated that peak stretch at failure is lower for samples from older patients [22]. It should be noted that no significant anisotropy was reported in these last three works.

The objectives of the present study are two-fold. First, to provide researchers with basic mechanical data on healthy and pathological (aneurysmal and BAV arterial tissues) ascending aortic wall measured in vitro at physiological temperature. Second, to evaluate the effect of the two pathologies on the mechanical deterioration and mechanical performance of the ascending aortic wall. The assessment of data of young healthy tissues (i.e. 16-35 years old) and the analysis of the anisotropic response of control and pathological tissues are both original contributions of this research.

\section{Materials and methods}

Healthy ascending aortic segments were obtained from patients who had died from non-cardiovascular-related causes at the Hospital Puerta de Hierro de Madrid. Aortic wall samples were obtained from 23 brain-dead heart donors, previously deemed acceptable for transplantation, aged between 16 and 57 . In all cases, the tissues were obtained according to a protocol approved by the Hospital Ethics Committee, which included the informed consent of the next of kin. Despite their origin, histological analyses were performed on samples from all segments to corroborate the absence of vascular pathologies. For the study, healthy specimens were divided into two groups depending on age: Group 0A ( $<35$ years old, nine patients, $25 \pm 3$ years) and Group 0B ( $>35$ years old, 14 patients, $51 \pm 2$ years). Donor information (sex, age and body surface area) is summarised in Table 1.

Pathologic tissue was obtained from patients undergoing ascending aorta surgery with or without aortic valve replacement at the Hospital Puerta de Hierro de Madrid. According to the protocol approved by the Hospital Ethics Committee, informed consent from the patients was obtained before the procedure. Ascending aortic samples were classified into two groups according to the presence/absence of a BAV. Group I enclosed segments coming from 12 patients diagnosed with BAV, aged between 36 and 80 years ( $57 \pm 5$ years).

Group II was made up of 14 patients aged between 44 and 81 (60 \pm 4 years) with normal (tricuspid) aortic valve and diagnosed with ascending aortic aneurysm. Five patients were operated due to ascending aortic thoracic aneurysm (AATA) and four had aortic insufficiency (AI) 
Table 1 Donor's data

\begin{tabular}{|c|c|c|}
\hline $\operatorname{Sex}(\mathrm{F}$ or $\mathrm{M})$ & Age (years) & BSA $\left(\mathrm{m}^{2}\right)$ \\
\hline \multicolumn{3}{|c|}{ Control group $0 \mathrm{~A}$ ( $<35$ years, 9 donors, $25 \pm 3$ years $)$} \\
\hline $\mathrm{F}$ & 31 & 1.78 \\
\hline M & 16 & 1.88 \\
\hline M & 35 & 1.82 \\
\hline M & 20 & 2.05 \\
\hline M & 18 & 1.98 \\
\hline M & 25 & 2.12 \\
\hline M & 20 & 1.54 \\
\hline M & 29 & 1.99 \\
\hline M & 32 & 1.95 \\
\hline \multicolumn{3}{|c|}{ Control group 0B ( $>35$ years, 12 donors, $52 \pm 2$ years) } \\
\hline M & 45 & 1.97 \\
\hline M & 49 & 1.93 \\
\hline $\mathrm{M}$ & 52 & 1.82 \\
\hline $\mathrm{F}$ & 50 & 1.78 \\
\hline $\mathrm{F}$ & 45 & 1.60 \\
\hline M & 50 & 1.81 \\
\hline $\mathrm{F}$ & 48 & 1.44 \\
\hline $\mathrm{M}$ & 57 & 2.06 \\
\hline M & 57 & 1.68 \\
\hline $\mathrm{F}$ & 46 & 1.66 \\
\hline $\mathrm{F}$ & 64 & 1.78 \\
\hline M & 62 & 1.86 \\
\hline
\end{tabular}

$F$ female, $M$ male, $B S A$ body surface area

together with AATA. Three patients underwent surgery having aortic valve (AoVR) and ascending aorta replacement, and two patients were heart receptors due to dilated cardiomyopathy (DCM) in conjunction with AATA. Patients in group II had neither Marfan's nor Loeys-Dietz's syndromes and only one case presented few atheroma plaques (Ather). Nevertheless, in that case the testing samples were taken far (at least $2 \mathrm{~cm}$ ) from the plaques and no significant differences were observed between the specimens excised closer or further from the plaques nor with the other patients from group II. Patient information of groups I and II is shown in Table 2.

All aortic samples were obtained at least $5 \mathrm{~mm}$ above the sinotubular junction and preserved in saline solution at $4{ }^{\circ} \mathrm{C}$ until testing within $24 \mathrm{~h}$ from excision. The average dimensions of the vessels studied in this work are summarised in Table 3.

\subsection{Test specimens}

Uniaxial tensile tests were performed in order to characterise the mechanical behaviour of the arterial wall. Dogbone shaped specimens (Fig. 1) with a central zone $10 \mathrm{~mm}$ long $\times 2 \mathrm{~mm}$ wide were stamped out from opened-up
Table 2 Patient's data

\begin{tabular}{|c|c|c|}
\hline $\operatorname{Sex}(\mathrm{F}$ or $\mathrm{M})$ & Age (years) & BSA $\left(\mathrm{m}^{2}\right)$ \\
\hline \multicolumn{3}{|c|}{ Bicuspid aortic valve group I (11 patients, $55 \pm 4$ years) } \\
\hline $\mathrm{F}$ & 36 & 1.72 \\
\hline M & 49 & 2.04 \\
\hline M & 63 & 1.76 \\
\hline $\mathrm{F}$ & 58 & 1.80 \\
\hline M & 48 & 1.80 \\
\hline M & 43 & 2.12 \\
\hline M & 75 & 1.79 \\
\hline M & 65 & 1.90 \\
\hline $\mathrm{F}$ & 63 & 1.68 \\
\hline M & 42 & 1.94 \\
\hline $\mathrm{F}$ & 66 & 1.87 \\
\hline \multicolumn{3}{|c|}{ Aneurysm group II (11 patients, $56 \pm 2$ years) } \\
\hline $\mathrm{F}$ & 57 & 1.71 \\
\hline M & 60 & 1.83 \\
\hline $\mathrm{F}$ & 45 & 1.95 \\
\hline M & 62 & 1.94 \\
\hline M & 44 & 1.95 \\
\hline M & 51 & 1.68 \\
\hline M & 58 & 1.97 \\
\hline M & 53 & 1.80 \\
\hline M & 71 & 2.12 \\
\hline M & 65 & 1.90 \\
\hline M & 51 & 1.94 \\
\hline
\end{tabular}

$F$ female, $M$ male, $B S A$ body surface area

Table 3 Average dimensions of ascending aortas for different groups

\begin{tabular}{lll}
\hline & Diameter $(\mathrm{mm})$ & Thickness $(\mathrm{mm})$ \\
\hline Control group 0A & $22.0 \pm 2.2$ & $1.9 \pm 0.3$ \\
Control group 0B & $23.7 \pm 4.4$ & $2.2 \pm 0.3$ \\
Bicuspid aortic valve group I & $38.0 \pm 2.0$ & $1.9 \pm 0.2$ \\
Aneurysm group I & $38.5 \pm 7.7$ & $2.0 \pm 0.3$ \\
\hline
\end{tabular}

aortas by means of metal dies of appropriate dimensions. To evaluate the anisotropy of aortic wall, two sets with at least three samples each were obtained in both, longitudinal and circumferential, directions for every aortic segment (Fig. 1). Specimen thickness was measured on each sample by means of a Mitutoyo 7301 thickness gage with $10 \mu \mathrm{m}$ accuracy. Initial thicknesses of descending aortic specimens ranged from 1.6 to $2.1 \mathrm{~mm}$.

\subsection{Experimental set up}

The specimens were mechanically and chemically (glued with cyanoacrylate) fixed to the grips of an 


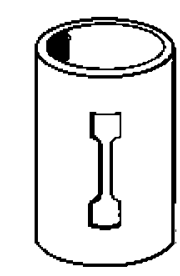

LONGITUDINAL

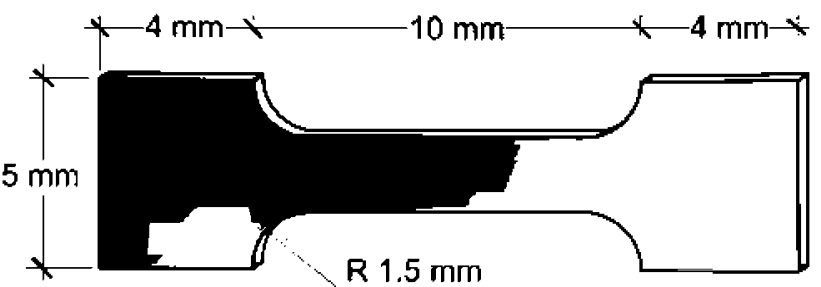

Fig. 1 Dimensions and orientation of aortic wall samples

electromechanical tensile testing machine (Instron 5866) and immersed in phosphate saline buffer (PBS) at $37^{\circ} \mathrm{C}$. The temperature of the vessel was controlled to $0.5^{\circ} \mathrm{C}$ by a $\mathrm{K}$-type thermocouple located in the chamber and close to the artery $(<4 \mathrm{~mm})$.

The axial force was measured by means of a $10 \mathrm{~N}$ load cell (Instron 2519-101, accuracy better than $5 \mathrm{mN}$ ) and the crosshead displacement was taken as a direct measurement of the elongation of the sample, as the compliance of the aortic specimen had been estimated as being 100 times greater than that of the equipment. The machine internal LVDT sensor (accuracy better than $10 \mu \mathrm{m}$ ) was used to measure the displacement of the crosshead.

All specimens were mechanically preconditioned by means of a series of five loading-unloading cycles between 0 and $30 \%$ of the maximum load, to remove the initial stress relaxation effect and to yield a stable response. Tests were performed in the passive state, without electrical or chemical activation of the vascular wall. Loading rate was $0.03 \mathrm{~mm} / \mathrm{s}$ (approximately $18 \% / \mathrm{min}$ strain rate). A detailed scheme of the testing procedure can be found elsewhere [11].

\subsection{Evaluation of the mechanical parameters}

True stress $(\sigma)$ versus stretch $(\hat{\lambda})$ relationships for all the tests were derived from experimental load-displacement curves. The stretch $(i)$ was obtained by dividing the current length $(l)$ by the initial length $(L)$ of the specimen, and the true stress $(\sigma)$ was calculated as the applied load $(P)$ divided by the current cross-sectional area $(a)$. The hypothesis of tissue incompressibility [18] was used to obtain the relationship between the current and the initial cross-sectional area $(A), a=A / l$.

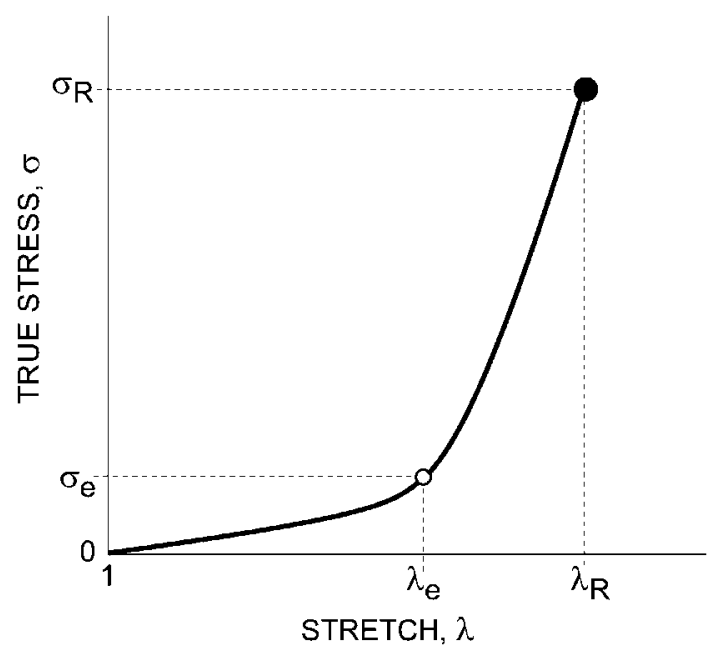

Fig. 2 Stress-stretch curve of aortic tissue and mechanical parameters

When tensile-tested, aortic wall specimens-either circumferential or longitudinal-typically display the characteristic $J$-shape curve depicted in Fig. 2. For small values of applied stress, the aortic tissue exhibits a compliant behaviour that turns into a much stiffer response when stresses exceed the elbow of the curve. To simplify the analysis and allow comparison among different specimens, the stress-stretch curve was condensed into three parameters that summarised the main mechanical response of the arterial wall: the stretch and stress at the breaking point $\left(\hat{\lambda}_{\mathrm{R}}, \sigma_{\mathrm{R}}\right)$, and the stress at the transition point, or "elbow", between the compliant and the stiff regions $\left(\sigma_{\mathrm{e}}\right)$.

The breaking point of the sample $\left(\hat{\lambda}_{\mathrm{R}}, \sigma_{\mathrm{R}}\right)$ was defined as the first point where one of its layers fails, easily identified in the tensile curve by a sudden drop of the load. Although in the vascular biomechanics literature the ultimate tensile strength is usually taken as the stress at the rupture stage, in the measurements reported in this work the difference between these two definitions was $<5 \%$. Therefore, the values presented in this study are comparable to other published data. Moreover, the elbow stress $\left(\sigma_{\mathrm{e}}\right)$ was determined by the change in the first derivative of stress with respect to stretch, according to the procedure given in the "Appendix".

\subsection{Data processing and statistics}

At least six specimens were tensile-tested per individual, three in each of two directions (longitudinal and circumferential). All in all, 355 tests on ascending aortic tissue were carried out. Values of the three parameters defined above were averaged for each individual and orientation, and individual means averaged again for each study group and orientation. Data are presented as mean \pm standard 
error. An unpaired two-tailed Student's $t$ test was performed to compare mechanical parameters. Significance was assumed for $p$ as $<0.05$.

\section{Results}

\subsection{Aortic wall strength}

The tensile strengths of the ascending aortic wall for the two control groups $\mathrm{OA}$ and $\mathrm{OB}$ (young and old specimens, respectively), and the two groups of pathologies are considered in the study. Tensile stretches at failure are shown in Fig. 3. To evaluate the anisotropy of the arterial wall, circumferential and longitudinal values are displayed for every group in both figures. No distinction has been made between male and female specimens, as no significant differences between the two sexes have been found in the experiments.

Directional differences in mechanical behaviour were found for healthy specimens, groups $\mathrm{OA}$ and $\mathrm{OB}$, where the circumferential failure stresses were greater than the longitudinal ones $(2.18 \pm 0.24$ vs. $1.14 \pm 0.10 \mathrm{MPa}$, $p=0.001$ for group $0 \mathrm{~A}$ and $1.20 \pm 0.20$ vs. $0.66 \pm 0.07$ $\mathrm{MPa}, p=0.02$ for group $\mathrm{OB})$. A similar trend was observed for BAV and aneurismal groups, although only in the BAV group the difference was statistically significant $(1.23 \pm 0.15 \mathrm{MPa}$ circumferential vs. $0.84 \pm 0.10 \mathrm{MPa}$ longitudinal, $p=0.04$ for group I (BAV), and $1.19 \pm 0.13$ $\mathrm{MPa}$ circumferential vs. $0.88 \pm 0.12 \mathrm{MPa}$ longitudinal, $p=0.09$ for group II (aneurysm).

Values of stretch at failure, shown in Fig. 3, confirm the anisotropy of the young healthy specimens, group $0 \mathrm{~A}$, ( $2.35 \pm 0.10$ circumferential vs. $2.00 \pm 0.10$ longitudinal, $p=0.03$ ) and BAV aortic wall tissues, group I $(1.80 \pm 0.08$ circumferential vs. $1.58 \pm 0.06$ longitudinal, $p=0.04$ ), with the differences being non significant for the other groups, either healthy (OB) or diseased (II).

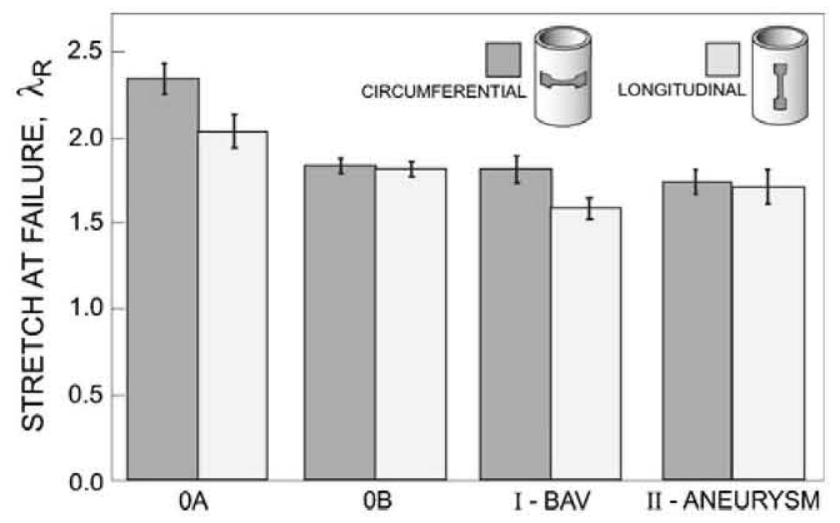

Fig. 3 Stretch at failure (mean \pm standard error) of ascending aortic wall samples for healthy and pathologic groups
To assess the risk of rupture of aortic ascending wall, tensile strengths have to be compared with the circumferential stresses exerted on the aortic wall by blood pressure, which can be readily evaluated to a good approximation by means of the thin-walled tube equation: $\sigma_{\theta}=$ $\frac{p}{2 t} \lambda_{\theta} \lambda_{z}\left(\lambda_{\theta} D-\frac{t}{\lambda_{\theta} \lambda_{z}}\right)$, where $\sigma_{\theta}$ is the circumferential stress, $p$ is the blood pressure, $\lambda_{\theta}$ is the circumferential stretch, $\lambda_{z}$ is the longitudinal stretch, $t$ the wall thickness and $D$ the aortic diameter. Note that $\sigma_{\theta}, p, \lambda_{\theta}, \lambda_{\mathrm{z}}$ are in vivo variables while $D, t$ are in vitro variables (see Table 3 ).

This equation allows the computation of the in vivo circumferential stress considering the following assumptions: (a) the longitudinal stretch under in vivo conditions is $\lambda_{z}=1.2$ [15] and (b) the circumferential stretch $\lambda_{\theta}$ is estimated from the tensile test measurements, as depicted in Fig. 4, resulting the values 1.52, 1.47, 1.44 and 1.38 for groups $0 \mathrm{~A}, 0 \mathrm{~B}, \mathrm{I}$ and II, respectively. Figure 5 shows the mean circumferential wall stresses computed for every group of study under the assumption of normotensive pressure levels $(100 \mathrm{mmHg})$. The in vivo ascending aortic diameters agree well with those reported in the literature for healthy specimens of corresponding age (around $30 \pm 0.5 \mathrm{~mm}$ ) [13] and for BAV group (50 $\pm 4 \mathrm{~mm}$ ) [19]. For aneurysm group (II), the computed in vivo circumferential wall stresses is similar to that measured elsewhere from a group of age-matched patients ( $65 \pm 5$ years) [17].

\subsection{Stress at the elbow of the tensile curve}

Tensile curves of aortic wall specimens display an initial compliant zone for small and medium strains followed by a region of higher stiffness. Elastin fibres contribute mostly to the first part of the curve, while progressive recruitment

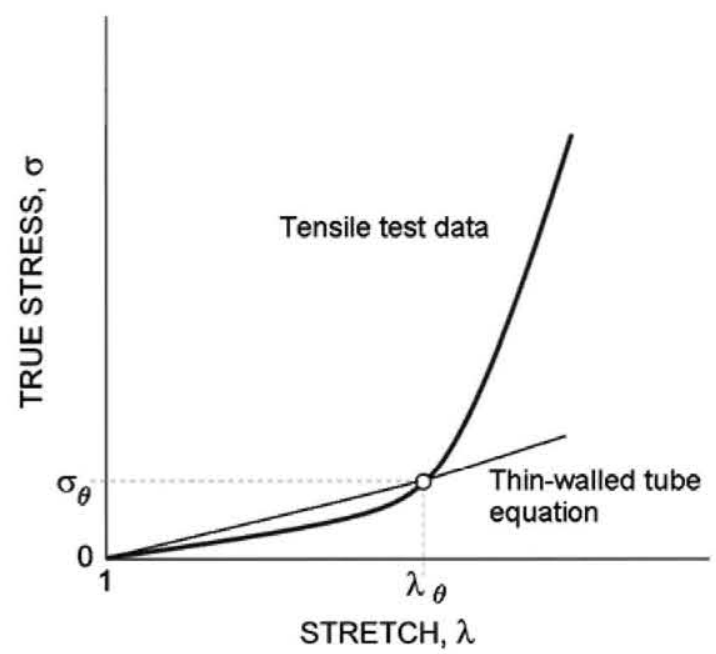

Fig. 4 Procedure to obtain the circumferential stretch for in vivo conditions 


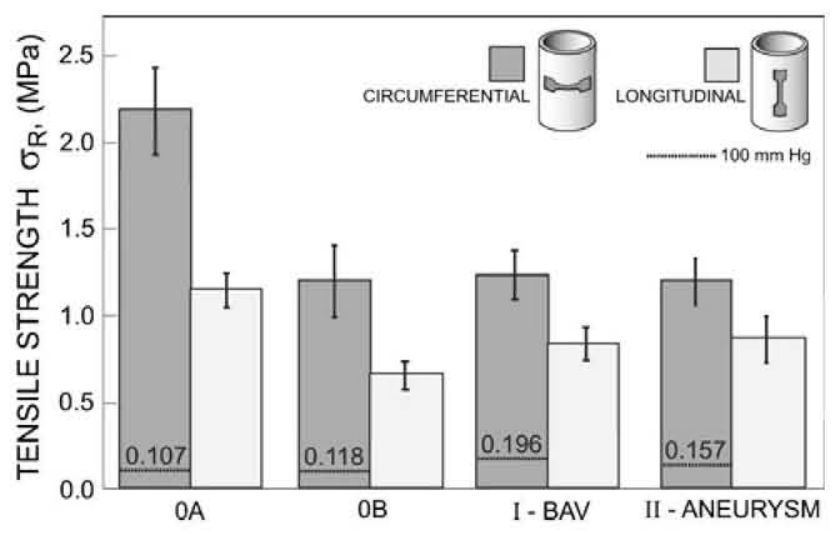

Fig. 5 Tensile strength (mean \pm standard error) of ascending aortic wall samples for healthy and pathologic groups. The mean physiological stress level in the aortic wall at $100 \mathrm{mmHg}$ is shown for each group

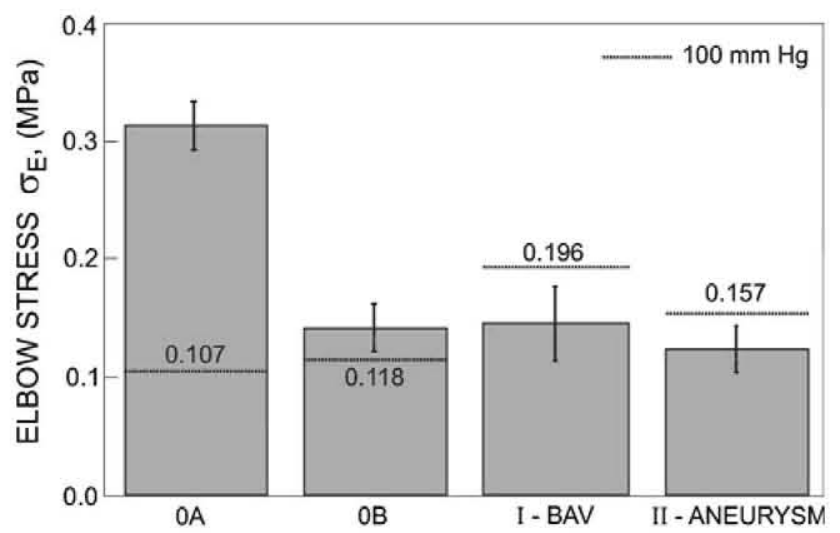

Fig. 6 Elbow stress (mean \pm standard error) of ascending aortic wall samples for healthy and pathologic groups. The mean physiological stress level in the aortic wall at $100 \mathrm{mmHg}$ is shown for each group

and extension of collagen fibres are responsible for the second region [21]. The transition zone, or elbow point, is usually well marked and can be characterised by its corresponding stress. Figure 6 plots the circumferential elbow stresses for the four groups of aortic specimens considered in this work. Elbow stresses were computed from stressstrain curves, as explained in the "Appendix". Figure 6 also shows the mean circumferential wall stresses produced by blood pressure $(100 \mathrm{mmHg})$ that were estimated earlier in this section.

The elbow stress decreases markedly with age, with it being more than halved in healthy specimens from $0.31 \pm 0.02 \mathrm{MPa}$ for group $0 \mathrm{~A} \quad(25 \pm 3$ years $)$ to $0.14 \pm 0.02 \mathrm{MPa}$ for group $0 \mathrm{~B}(51 \pm 2$ years). Pathologies do not show a subsequent reduction of elbow stress, as their values do not show significant differences with respect to group $0 \mathrm{~B}(0.15 \pm 0.02$ for group $\mathrm{I}, \mathrm{BAV}$, and $0.13 \pm 0.02 \mathrm{MPa}$ for group II, aneurysm).

\section{Discussion}

\subsection{Aortic wall strength}

Although our results are partially in agreement with previous data reported elsewhere [23], which did not find significant difference between circumferential and longitudinal specimens of either control or aneurismal tissue of aged patients, the trend noted in Fig. 5 suggests that a small anisotropy could be present in both diseased and healthy aortic walls, as has also been recently reported [16].

While the effect of age on the aortic wall strength had been noticed in dilated ascending aorta [19], such a phenomenon had not been demonstrated yet in healthy specimens. Figures 3 and 5 show that age has a predominant role in the mechanical behaviour of the healthy ascending aortic wall, as tensile mechanical resistance decreases markedly when age is doubled from group OA to OB. This effect is more pronounced in tensile strength that reduces in group $\mathrm{OB}$ in circumferential direction up to $55 \%$ of value corresponding to group OA. In contrast, circumferential stretch at failure falls only by $22 \%$ from the young to the older specimens. In longitudinal direction, older specimens retain up to 58 and $89 \%$ of the tensile strength and stretch at failure of younger ones, respectively, with these differences being statistically significant.

The effect of age is even more remarkable if, as noted in Figs. 3 and 5, the reduction of stress and stretch at failure due to aging places the mechanical parameters of healthy old specimens-either measured in circumferential or longitudinal directions - at a level statistically undistinguishable from pathologic aortas, with the only exception being the failure stretch of specimens from group I (BAV), which is significantly lower than the longitudinal stretch of group $0 \mathrm{~B}$. Other values of stretch at failure or tensile strength from group OB are statistically similar to BAV (I) or aneurysm (I) pathologic groups, even though the mean age of group $0 \mathrm{~B}$ is a little younger. Our results confirm recently published data on tensile strength of healthy and aneurysmal ascending aortic tissues [16] and stress, the importance of comparison of age-matched specimens to avoid biased conclusions.

Another interesting result deduced from Figs. 3 and 5 is that the effect of pathology makes no significant differences in rupture parameters, as tensile strengths and stretches at failure measured on tissues from patients of comparable ages were found statistically undistinguishable. The measured circumferential tensile strengths and stretches for groups I (BAV) and II (aneurysm) are concordant with the values reported elsewhere on dilated ascending aortas of age-matching patients [19].

Tensile strengths of ascending aortic tissues are much higher than wall stresses for all conditions, with factors of 
safety (defined as the ratio of tensile strength to the mean wall stress) about 20 for group 0A. Interestingly, while circumferential tensile strength seems to reduce to approximately the same stress levels for groups OB (old healthy), I (BAV) and II (aneurysm), the factor of safety shows noticeable differences between healthy and pathologic groups (10.2 for $0 \mathrm{~B}$, and 6.3 and 7.6 for I and II, respectively), thus reflecting the different working stress at the vessel wall.

\subsection{Mechanical performance of the ascending aortic wall}

The determination of the tensile strength is of unquestionable interest in evaluating the deterioration and risk of rupture of aortic wall. Nevertheless, the results shown previously from pathologic samples-that were obtained from patients undergoing aortic replacement-demonstrate that wall stresses due to the cardiac cycle lie fairly below the rupture level of aortic wall. In contrast, degeneration and dilatation of the aortic wall seems to be a continuous process caused, among other factors, by the permanent effect of alternating stresses due to blood pressure. From this perspective, the evaluation of the mechanical performance of the aortic tissue at the working point and its variation with age and pathology is of primary interest to cardiovascular research.

The circumferential stress level set off by blood pressure causes the aortic wall to work during the cardiac cycle, either in the compliant elastin-dominated part before the elbow or in the stiff collagen-dominated zone beyond it. Consequently, it is expected that variations in the mechanical behaviour of the aortic wall produced by age or pathologies lead to a change in the position of the elbow point and have a direct effect on the arterial compliance throughout the cardiac cycle.

When compared to the wall stresses produced by blood pressure, only the elbow stress of healthy specimens lies above, meaning that these aortas work in the initial compliant zone. The ratio between the circumferential wall stress and elbow stress is 0.34 for group $0 \mathrm{~A}$ and 0.83 for group $\mathrm{OB}$, with the older group being close to the upturning zone. For the pathologic groups the ratio grows to 1.33 and 1.26 (I and II, respectively), meaning that during physiological operation the aorta works in the stiffer part of its response curve, losing part of its function of damping the pressure waves from the cardiac beat. The reduction in distensibility is present in most pathologies, and connected to deterioration and destructive remodelling of the aortic wall $[9,16]$. Although altered distensibility alone cannot account for a higher risk of rupture, increases of in vivo stiffness overload collagen fibres and promote a loss of elastic fibres [16], enlarging aortic diameter and thereby raising the wall stress and indirectly influencing the risk of rupture. Moreover, reduced aortic elasticity and aortic root dilatation in non-stenotic BAV patients were previously in in vivo measurements [10].

In conclusion, the mechanical behaviour and strength of the human ascending aorta, both in healthy and pathological conditions, have been measured in this work through in vitro tensile tests. Results show that aging causes a significant decrease of rupture loads and elongations at breaking, comparable to the effect of severe pathologies such as aneurysm and BAV. While aortic ascending wall strength decreases significantly beyond the age of 35 , the mean physiological wall stresses acting on either healthy or pathologic aortas are always far from the rupture point, displaying factors of safety larger than six. In contrast, the physiological operation of pathologic vessels seems to be differentially affected by the disease since, contrary to the behaviour of healthy control aortas of similar age, patients with aneurysm and BAV have their ascending aortas working in the stiff part of its response curve, and

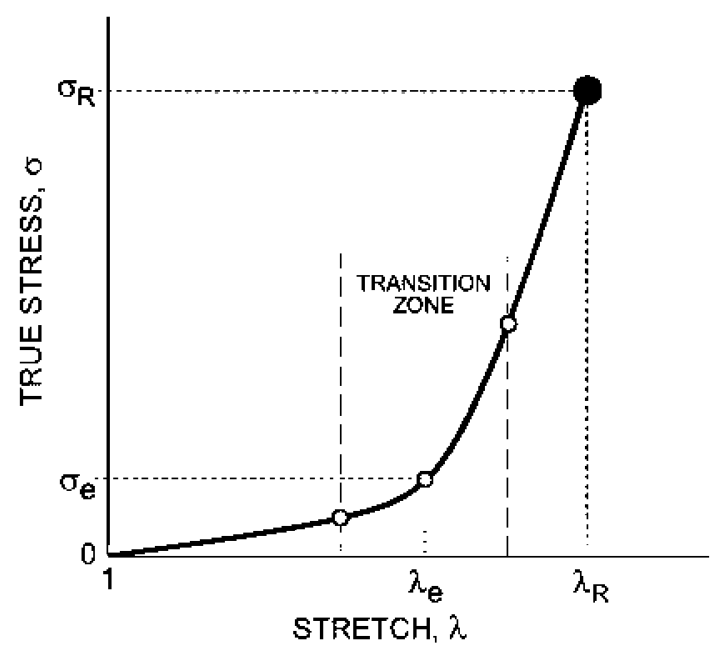

Fig. 7 Stress-stretch curve of the aortic wall

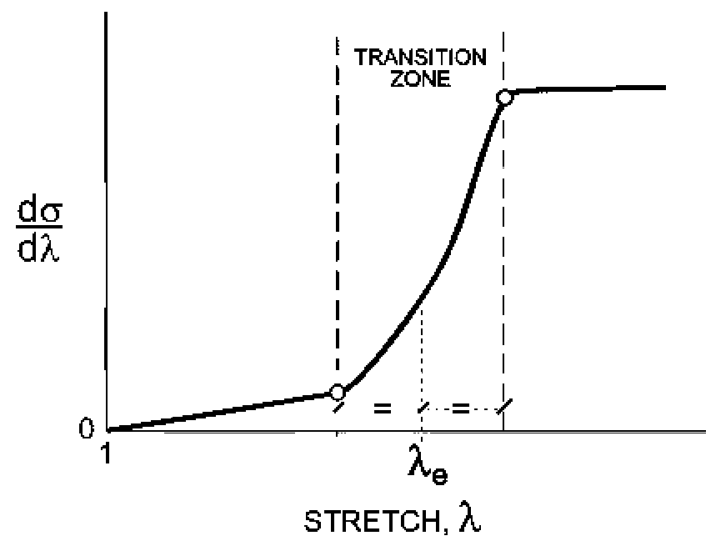

Fig. 8 First derivative of the stress-stretch curve 
consequently impairing the aortic elasticity. Finally, the role of biological processes on the mechanical behaviour of the aortic wall has not been considered in the present analysis and, therefore, this is an aspect that needs to be explored in future works.

Acknowledgments The authors wish to express their appreciation to Dr J. Goicolea of the Hospital de Puerta de Hierro at Madrid for the provision of arterial tissues analysed in this work. The support provided by the the FONDECYT Project No. 11090266 of the Chilean Council of Research and Technology (CONICYT) is all gratefully acknowledged.

\section{Appendix: Determination of the elbow point of the stress-stretch curve}

This appendix explains the method followed to obtain the coordinates of the transition point of the stress-stretch curve (Fig. 7). The procedure is based on the first derivative of the stress-stretch curve $(\mathrm{d} \sigma / \delta \lambda)$ (Fig. 8), that was obtained numerically for each specimen by means of the software "Kaleidagraph ${ }^{(\mathcal{)}}$,

On the $(\mathrm{d} \sigma / \delta \lambda)$ curve, the transition zone is readily identified as the nearly linear central region before the upper plateau, as shown in Fig. 8. The elbow point is defined in this work as the mid-point of the transition zone (Fig. 8). The stretch at the elbow $\left(\lambda_{\mathrm{e}}\right)$ is then determined on the $(\mathrm{d} \sigma / \delta 1)$ curve and the corresponding stress $\left(\sigma_{\mathrm{e}}\right)$ is obtained directly from the stress-stretch curve.

\section{References}

1. Bonow RO, Carabello BA, Chatterjee K, de Leon AC Jr, Faxon DP, Freed MD, Gaasch WH, Lytle BW, Nishimura RA, O'Gara PT, O'Rourke RA, Otto CM, Shah PM, Shanewise JS, Smith SC Jr, Jacobs AK, Adams CD, Anderson JL, Antman EM, Faxon DP, Fuster V, Halperin JL, Hiratzka LF, Hunt SA, Lytle BW, Nishimura R, Page RL, Riegel B (2006) ACC/AHA 2006 Guidelines for the management of patients with valvular heart disease: a report of the American College of Cardiology/American Heart Association Task Force on practice guidelines (writing committee to revise the 1998 guidelines for the management of patients with valvular heart disease) developed in collaboration with the society of cardiovascular anesthesiologists endorsed by the society for cardiovascular angiography and interventions and the society of thoracic surgeons. J Am Coll Cardiol 48(3):e1-e148

2. Boudoulas H, Toutouzas P, Wooley C (1996) Functional abnormalities of the aorta. Futura, Armonk

3. Coady MA, Rizzo JA, Goldstein LJ, Elefteriades JA (1999) Natural history, pathogenesis, and etiology of thoracic aortic aneurysms and dissections. Cardiol Clin 17(4):615-635

4. Darling RC, Messina CR, Brewster DC, Ottinger LW (1977) Autopsy study of unoperated abdominal aortic-aneurysms: case for early resection. Circulation 56(3):161-164

5. Davies RR, Goldstein LJ, Coady MA, Tittle SL, Rizzo JA, Kopf GS, Elefteriades JA (2002) Yearly rupture or dissection rates for thoracic aortic aneurysms: simple prediction based on size. Ann Thorac Surg 73(1): 17-28

6. Davies RR, Gallo A, Coady MA, Tellides G, Botta DM, Burke B, Coe MP, Kopf GS, Elefteriades JA (2006) Novel measurement of relative aortic size predicts rupture of thoracic aortic aneurysms. Ann Thorac Surg 81(1):169-177

7. Erbel R, Eggebrecht H (2006) Dimensions and the risk of dissection. Heart 92(1): 137-142

8. Fedak PWM, Verma S, David TE, Leask RL, Weisel RD, Butany J (2002) Clinical and pathophysiological implications of a bicuspid aortic valve. Circulation 106(8):900-904

9. Groenink M, Langerak SE, Vanbavel E, van der Wall EE, Mulder BJM, van der Wal AC, Spaan JAE (1999) The influence of aging and aortic stiffness on permanent dilation and breaking stress of the thoracic descending aorta. Cardiovase Res 43(2):471-480

10. Grotenhuis HB, Ottenkamp J, Westenberg JJM, Bax JJ, Kroft LJM, Roos A (2007) Reduced aortic elasticity and dilatation are associated with aortic regurgitation and left ventricular hyperthrophy in nonstenotic bicuspid aortic valve patients. J Am Coll Cardiol 49:1660-1665

11. Guinea GV, Atienza JM, Elices M, Aragoncillo P, Hayashi K (2005) Thermomechanical behavior of human carotid arteries in the passive state. Am J Physiol Heart Circ Physiol 288(6):H2940-H2945

12. Guntheroth WG (2008) A critical review of the American College of Cardiology/American Heart Association practice guidelines on bicuspid aortic valve with dilated ascending aorta. Am J Cardiol 102(1): $107-110$

13. Hager A, Kaemmerer H, Rapp-Bernhardt U, Blucher S, Rapp K. Bernhardt TM, Galanski M, Hess J (2002) Diameters of the thoracic aorta throughout life as measured with helical computed tomography. J Thorac Cardiovasc Surg 123(6):1060-1066

14. Hall AJ, Busse EFG, McCarville DJ, Burgess JJ (2000) Aortic wall tension as a predictive factor for abdominal aortic aneurysm rupture: improving the selection of patients for abdominal aortic aneurysm repair. Ann Vasc Surg 14(2):152-157

15. Humphrey JD (2001) Cardiovascular solid mechanics: Cells, Tissues and Organs. Springer

16. Iliopoulos DC, Kritharis EP, Giagini AT, Papadodima SA, Sokolis DP (2009) Ascending thoracic aortic aneurysms are associated with compositional remodeling and vessel stiffening but not weakening in age-matched subjects. J Thorac Cardiovasc Surg 137(1):101-109

17. Koullias G, Modak R, Tranquilli M, Korkolis DP, Barash P, Elefteriades JA (2005) Mechanical deterioration underlies malignant behavior of aneurysmal human ascending aorta. J Thorac Cardiovase Surg 130(3):677.e1-677.e9

18. Oijen CV (2003) Mechanics and design of fiber-reinforced vascular prostheses ( $\mathrm{PhD}$ thesis). Technische Universiteit Eindhoiven

19. Okamoto RJ, Wagenseil JE, DeLong WR, Peterson SJ, Kouchoukos NT, Sundt TM (2002) Mechanical properties of dilated human ascending aorta. Ann Biomed Eng 30(5):624-635

20. Rizzo JA, Coady MA, Elefteriades JA (1999) Interpreting data on thoracic aortic aneurysms. Statistical issues. Cardiol Clin 17(4):797-805

21. Roach MR, Burton AC (1957) The reason for the shape of the distensibility curves of arteries. Can J Biochem Physiol. 35(8):681-690

22. Vande Geest JP, Di Martino ES, Vorp DA (2004) An analysis of the complete strain field within Flexcercell ${ }^{\mathrm{TM}}$ membranes. J Biomech 37:1923-1928

23. Vorp DA, Schiro BJ, Ehrlich MP, Juvonen TS, Ergin MA, Griffith BP (2003) Effect of aneurysm on the tensile strength and biomechanical behavior of the ascending thoracic aorta. Ann Thorac Surg 75(4):1210-1214

24. Vorp DA, Vande Geest JP (2005) Biomechanical determinants of abdominal aortic aneurysm rupture. Arterioscler Thromb Vasc Biol 25(8):1558-1566 